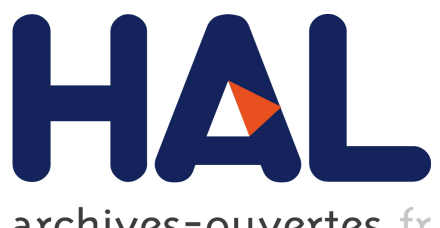

archives-ouvertes

\title{
Contextual Edit Distance for Semantic Trajectories
}

Clément Moreau, Thomas Devogele, Veronika Peralta, Laurent Etienne

\section{To cite this version:}

Clément Moreau, Thomas Devogele, Veronika Peralta, Laurent Etienne. Contextual Edit Distance for Semantic Trajectories. ACM Symposium On Applied Computing, Mar 2020, Brno, Czech Republic. 2020, 10.1145/3341105.3374125 . hal-02382303

\section{HAL Id: hal-02382303 \\ https://hal.archives-ouvertes.fr/hal-02382303}

Submitted on 5 Apr 2021

HAL is a multi-disciplinary open access archive for the deposit and dissemination of scientific research documents, whether they are published or not. The documents may come from teaching and research institutions in France or abroad, or from public or private research centers.
L'archive ouverte pluridisciplinaire HAL, est destinée au dépôt et à la diffusion de documents scientifiques de niveau recherche, publiés ou non, émanant des établissements d'enseignement et de recherche français ou étrangers, des laboratoires publics ou privés. 


\title{
A Contextual Edit Distance for Semantic Trajectories
}

\author{
Clement Moreau, Thomas Devogele, Veronika Peralta and Laurent Etienne \\ University of Tours \\ Blois, France \\ firstname.lastname@univ-tours.fr
}

\begin{abstract}
The understanding of daily human activity is an active research topic. Thanks to GPS and smartphones, human movements can be monitored and analyzed. In addition, by exploiting Linked Open Data and user personal data, semantic labels and annotations can be added to movements. Thus, semantic trajectories can be considered as sequences of timestamped activities where each activity is described by a semantic label. In this context, a major challenge is the comparison of such semantic trajectories, looking to extract and learning similar human mobility behaviors. We propose $C E D$ (Contextual Edit Distance), a generic similarity measure for semantic sequences comparison which improve the Edit Distance to take into account the context similarity between elements in the sequence. CED is configurable to any sequence data and business needs.
\end{abstract}

\section{CCS CONCEPTS}

- Information systems $\rightarrow$ Similarity measures; Geographic information systems.

\section{KEYWORDS}

edit distance, semantic trajectory, human behavior analysis, similarity measure, clustering

\section{ACM Reference Format:}

Clement Moreau, Thomas Devogele, Veronika Peralta and Laurent Etienne. 2020. A Contextual Edit Distance for Semantic Trajectories. In The 35th ACM/SIGAPP Symposium on Applied Computing (SAC '20), March 30-April 3, 2020, Brno, Czech Republic. ACM, New York, NY, USA, 3 pages. https: //doi.org/10.1145/3341105.3374125

\section{INTRODUCTION}

Semantic trajectories enrich the classical trajectories (sequences of space-time positions [7]) with semantic labels representing activities [11], POI [5] or sets of aspects [1, 3, 8]. They allow to model richer aspects of human behavior that were not captured by traditional trajectories. In particular, they allow the semantic comparison of trajectories, in order to capture frequent behavior, detect atypical moves, and more generally, understand human activity. For example, from a sociological point of view, two trajectories representing two people' moves "home-football" and "home-basketball" may represent similar behavior (trips to play sport), even if they live at different neighbourhoods and move at different times. Therefore, similarity measures, which are at the core of several mobility data mining methods, should take advantage of semantic labels in the comparison of semantic trajectories. There exists many measures to compare semantic trajectories like $[3,4,6]$. However, They generally use trivial distances (e.g binary, euclidean) between symbols of the sequences. Moreover, they do not take into account the notion of context to compare the sequences. We understand the term context as the semantic content, or a portion, of the sequence (i.e. trajectory).

In this paper we present a new distance for combaring semantic

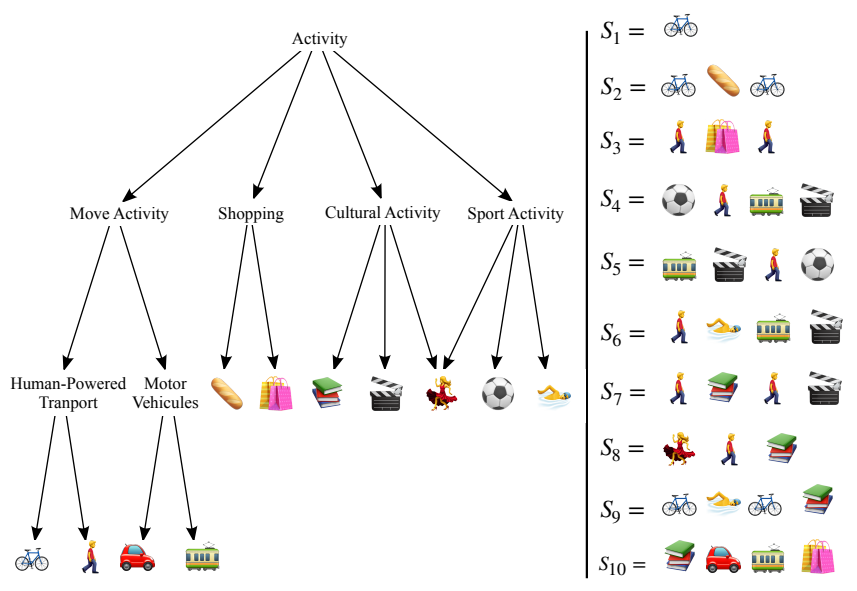

Figure 1: Ten semantic trajectories and the associated concept hierarchy based on an simple ontology

In the rest of this paper, we note $\Sigma$ a set of symbols e.g or $S \in \Sigma^{n}$ is a finite sequence of symbols, $|S|=n$ denotes the length of sequence $S$. The main interest of CED is the three following properties:

Property 1 (SEmAntic SIMILARITy). We consider there exists a similarity metric sim : $\Sigma \times \Sigma \rightarrow[0,1]$ between symbols and quantify their semantic proximity. This sim measure can be obtained by building a directed acyclic graph (e.g. a concept hierarchy based on an ontology) on $\Sigma$ 's elements [2].

Property 2 (Contextual Semantic edition). Given a semantic sequence $S \in \Sigma^{n}$ and a symbol $a \in \Sigma$ to edit in $S$. The edition cost of a depends on the similarity of nearby symbols in $S$. 
For example, similarity between $S_{7}$ and $S_{8}$ is high. Indeed, the main difference is the presence of $\in S_{7}$ and $\in S_{8}$. Nevertheless, theses two cultural activities are closed.

Property 3 (Repetition AND Permutation). Given a semantic sequence $S \in \Sigma^{n}$ and a symbol $a \in \Sigma$ to edit in $S$. Edition or permutations of repeated close symbols has little cost.

For example, similarity between $S_{1}$ and $S_{2}$ is good enough. Indeed, Although $S_{4}, S_{5}$ and $S_{6}$ are made up of the same or close symbols but swapped, so we can say they are similar.

Contribution. In this paper we present a new similarity measure inspired by the Edit Distance, the Contextual Edit Distance (CED), for semantic sequences apply on semantic trajectories. This measure focus on the semantic dimension and takes into account the similarity between symbols of the sequence and the context. The remainder of this article is organized as follows. Section 2 mathematically describes the CED while Section 3 presents a simple clustering process to illustrate the advantages of this measure. Section 4 concludes the paper.

\section{CONTEXTUAL EDIT DISTANCE}

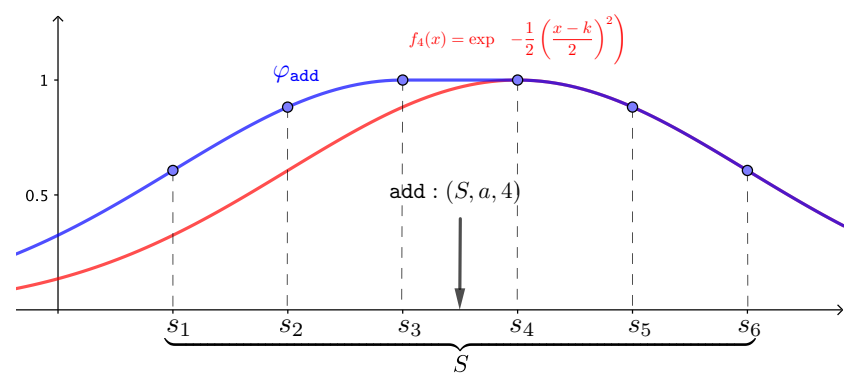

Figure 2: Add a symbol $a$ at position 4 in the sequence $S$

Definition 1 (Contextual edit operation). A contextual edit operation e is a function $e:\left(\Sigma^{*} \times \Sigma \cup\{\varepsilon\} \times \mathbb{N}\right) \rightarrow \Sigma^{*}$ whose arguments are a sequence, a new symbol to be included in the sequence (or none) and the index (position) in the sequence where the edition takes place.

We consider the following set $O=\{\mathrm{mod}, \mathrm{add}, \mathrm{del}\}$ of contextual edit operations:

- $\bmod :(S, a, k) \mapsto\left\langle s_{1}, \ldots, s_{k-1}, a, s_{k+1}, \ldots, s_{n}\right\rangle$ Replace the symbol at index $k$ by the new symbol $a$.

- add : $(S, a, k) \mapsto\left\langle s_{1}, \ldots, s_{k-1}, a, s_{k}, \ldots, s_{n}\right\rangle$ Insert symbol $a$ at index $k$. The symbols at and after index $k$ are shifted forward.

- del : $(e, \varepsilon, k) \mapsto\left\langle s_{1}, \ldots, s_{k-1}, s_{k+1}, \ldots, s_{n}\right\rangle$ Delete the symbol at position $k$. The symbols after position $k$ are shifted backward.

In order to take into account duplication, contextual information and permutation, we introduce the notion of context vector and context function that control the level of temporal proximity of two activities. Thus, thanks to a context vector, relationship factor is computed.

Definition 2 (CONTEXT FUnCTION). Consider a contextual edit operation $e(S, a, k)$ and a function $f_{k}: \mathbb{R} \rightarrow[0,1]$ which holds the following properties:

(1) $f_{k}(k)=1$.

(2) $f_{k}$ is a monotonically increasing function on $\left.]-\infty, k\right]$.

(3) $f_{k}$ is symmetrical center on $k$.

The third property guarantees to take with the same importance previous and future symbols located at equal distance from $s_{k}$.

We denote $f_{k}$ as context function. A context edit function $\varphi_{e}$ : $\mathbb{N}^{*} \rightarrow[0,1]$ is a transformation of $f_{k}$ stretching the function according to the type of operation e. We distinguish three cases for $e$ in \{mod, add, del\}:

- $\varphi_{\text {mod }}(x)=f_{k}(x)$

- $\varphi_{\text {add }}(x)= \begin{cases}f_{k}(x+1) & \text { if } x \leq k-1 \\ f_{k}(x) & \text { if } x \geq k\end{cases}$

- $\varphi_{\text {del }}(x)= \begin{cases}f_{k}(x+1) & \text { if } x \leq k-1 \\ 0 & \text { if } x=k \\ f_{k}(x-1) & \text { if } x \geq k+1\end{cases}$

Finally, a context vector $v: E \rightarrow[0,1]^{n}$ is defined as

$$
v(e)=\left\langle v_{1}, \ldots, v_{n}\right\rangle
$$

where $v_{i}=\varphi_{e}(i)$

Intuitively, the context vector quantifies the relationship between symbol $s_{k}$ and another symbol $s_{i}$. Bigger $|k-i|$ is, lesser the symbols $s_{i}$ has an impact on $s_{k}$. Thus, the relationship factor weighted the similarity values between two symbols.

The cost function $\gamma$ of CED generalizes the classical definition of Edit Distance and takes into account the local context of each query in the exploration.

Definition 1 (Cost function $\gamma)$. Given an operator $e(S, a, k)$ a cost function $\gamma: E \rightarrow[0,1]$ for the contextual edit operations is defined as:

$$
\gamma(e)=\alpha \times \delta(e)+(1-\alpha)\left(1-\max _{i \in \llbracket 1, n \rrbracket}\left\{\operatorname{sim}\left(s_{i}, a\right) \times v_{i}(e)\right\}\right)
$$

where:

- $\alpha \in[0,1]$ is a contextual parameter.

If $\alpha \rightarrow 0$ the contextual part is maximal and therefore the distance between two queries will be strongly evaluated according to the content of the sequence being edited ; if $\alpha \rightarrow 1$ then cost of edition is fixed and CED is equivalent to Edit Distance.

- $\delta(e)= \begin{cases}1-\operatorname{sim}\left(s_{k}, x\right) & \text { if } e=\bmod \\ 1 & \text { else }\end{cases}$

is the local cost of the Edit Distance.

Definition 2 (Edit path). Given two sequences $S, S^{\prime} \in \Sigma^{*}$, an edit path $P=\left\langle e_{1}, e_{2}, \ldots, e_{m}\right\rangle$ from $S$ to $S^{\prime}$ is a sequence of operations that transform $S$ in $S^{\prime}$. We note $\mathcal{P}\left(S, S^{\prime}\right)$ the set of all edit paths to transform $S$ in $S^{\prime}$. 
An important point to be mentioned here is that, for some reasons of complexity and computation time, the calculation of context vector is based on the start sequence $S$ i.e $S^{(0)}$. Moreover, there is an asymmetric relation between elements due to the fact that add operator is not the reverse operator of del and vice versa.

Thus, by re-using the classic definition of the Edit Distance [9], we can define the one-side distance from $S_{1}$ to $S_{2}$.

Definition 3 (One-sided Contextual Edit Distance). Let $\tilde{d}_{C E D}$ : $\Sigma^{n} \times \Sigma^{p} \rightarrow \mathbb{R}^{+}$be the Contextual Edit Distance from $S_{1}$ to $S_{2}$ such that:

$$
\tilde{d}_{C E D}\left(S_{1}, S_{2}\right)=\min _{P \in \mathcal{P}\left(S_{1}, S_{2}\right)}\left\{\sum_{i=1}^{|P|} \gamma\left(e_{i}\right)\right\}
$$

To save the symmetry, we use the same trick as Hausdorff distance and we apply the max operator on each one-sided contextual edit distance.

Definition 4 (Contextual Edit Distance). Let $d_{C E D}: \Sigma^{n} \times$ $\Sigma^{p} \rightarrow \mathbb{R}^{+}$be the Contextual Edit Distance such that:

$$
d_{C E D}\left(S_{1}, S_{2}\right)=\max \left\{\tilde{d}_{C E D}\left(S_{1}, S_{2}\right), \tilde{d}_{C E D}\left(S_{2}, S_{1}\right)\right\}
$$

About time complexity, we re-use Wagner and Fisher algorithm and dynamic programming approach [9]. This guarantees CED a polynomial complexity in $O(n \times p \times \max (n, p))$.

\section{RUNNING EXAMPLE}

To compute the similarity between concepts for this running example, we choose the Wu-Palmer similarity [10]. The main goal of our experiments is to compare CED and Edit Distance results for trajectories having several degrees of similarities and confirm properties in section 2. The dendogram produced with Edit Distance is a baseline for understanding the interest and properties of CED. Figure 3 shows the resulting dendrograms with Ward agglomeration criteria performed in Python. To obtain this dendrogram, we set up CED with $\alpha=0$ and the same context function $f_{k}$ show in Figure 2. We highlight several interesting results of the analysis of CED values between some sets of sequences of activities . First, the contextual information is effectively used and has an impact in the computation of CED. For example, $S_{7}, S_{8}$ have a similar context : Two cultural activities and walking. And, indeed $d_{C E D}\left(S_{7}, S_{8}\right)<d_{E D}\left(S_{7}, S_{8}\right)$. We assume that this couple is correctly grouped together with CED. In addition, if an activity is split by a new activity, only the cost of adding this new activity is added. We can notice this property on $S_{6}, S_{7}$ but also on the cluster $\left\{S_{1}, S_{2}, S_{3}\right\}$ with $S_{1}$ and $S_{2}$. Finally, the cluster $\left\{S_{4}, S_{5}, S_{6}\right\}$ is composed by sequences with same (or close e.g. foot, swim) elements but with different order. This set confirms the permutation property. Globally, CED changes the topology of the space by bringing together sequences with similar content. To summarize, using CED as similarity measure significantly improves the clustering process of theses semantic trajectories according to properties claim in introduction. In the same way, CED improves outlier detection processes.

\section{CONCLUSION}

This paper addressed the problem of comparing semantic trajectories, which is a hot topic for the understanding of human behavior. Concretely, it defines a new measure, CED, to compute the

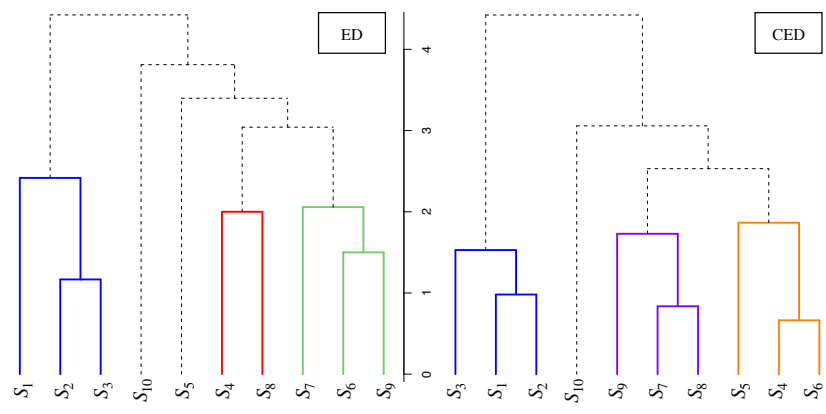

Figure 3: Hierarchical clustering based on (a) Edit Distance and $(b)$ CED. Cut based on the higher relative loss of inertia.

distance between semantic sequences. This measure extends the Edit Distance and its operations by taking context into account. It uses ontologies to describe symbols and to calculate the similarity between them. Thus, new aspects are taken into account as semantic similarity, local context of each activity and permutations. Moreover, the polynomial complexity of our algorithm (based on Dynamic Programming) gives hope to process large data sets.

In near future, CED will be tested on two large data sets concerning two real application domains: tourism circuits for recommendation and children daily mobility for clustering behaviour. We would also consider time aspect of symbol in order to express, for example, the duration of an activity. Furthermore, we will investigate other domains of application of CED, for example to measure the contextual similarity between log files.

\section{ACKNOWLEDGMENTS}

This project is funded by the ANR Mobi'kids and the CVL region (FRANCE).

\section{REFERENCES}

[1] V. Bogorny, C. Renso, A. Ribeiro de Aquino, F. de Lucca Siqueira, and LO. Alvares. 2014. CONSTAnT - A Conceptual Data Model for Semantic Trajectories of Moving Objects. Transactions in GIS 18 (2014), 66-88.

[2] M. M. Deza and E. Deza. 2016. Encyclopedia of Distances. Springer, Chapter 22 Distances in Internet and Similar Networks.

[3] R. dos Santos Mello, V. Bogorny, LO. Alvares, L.HZ Santana, CA. Ferrero, AA. Frozza, GA. Schreiner, and C. Renso. 2019. MASTER: A multiple aspect view on trajectories. Transactions in GIS (2019), 1-20.

[4] AS. Furtado, D. Kopanaki, LO. Alvares, and V. Bogorny. 2016. Multidimensional Similarity Measuring for Semantic Trajectories. Transactions in GIS 20 (2016), 280-298.

[5] F. Gianotti, M. Nanni, D. Pedreschi, and F. Pinelli. 2007. Trajectory Pattern Mining. ACM SIGKDD (2007), 330-339.

[6] A.L. Lehmann, L.O. Alvares, and V. Bogorny. 2019. SMSM: a similarity measure for trajectory stops and moves. IFGIS 33, 9 (2019), 1847-1872.

[7] C. Parent, S. Spaccapietra, C. Renso, G. Andrienko, V. Bogorny, ML. Damiani, A. Gkoulalas-Divanis, JA. de Macedo, N. Pelekis, Y. Theodoridis, and Z. Yan. 2013. Semantic trajectories modeling and analysis. Comput. Surveys 45 (2013), 1-32.

[8] LM. Petry, CA. Ferrero, LO. Alvares, C. Renso, and V. Bogorny. 2019. Towards Semantic-Aware Multiple-Aspect Trajectory Similarity Measuring. Transactions in GIS (2019).

[9] R. Wagner and M. Fisher. 1974. The String-to-String Correction Problem. F. ACM 21 (1974), 168-173.

[10] Z. Wu and M. Palmer. 1994. Verbs semantics and lexical selection. In Proceedings of the 32nd annual meeting on Association for Computational Linguistics. 133-138.

[11] C. Zhang, J. Han, L. Shou, J. Lu, and T. La Porta. 2014. Splitter: Mining FineGrained Sequential Patterns in Semantic Trajectories. VLDB Endowment 7 (2014), 769-780. 